\title{
A Hypersingular Boundary Integral Equation for a Class of Problems Concerning Infiltration from Periodic Channels
}

\author{
David L. Clements \\ Department of Applied Mathematics \\ The University of Adelaide \\ SA 5005, Australia \\ Maria Lobo \\ Department of Applied Mathematics \\ The University of Adelaide \\ SA 5005, Australia \\ Nyoman Widana \\ Department of Mathematics \\ Universitas Udayana \\ Bali, Indonesia
}

\begin{abstract}
In this paper some two dimensional infiltration problems are considered. The problems involve infiltration from periodic irrigation channels into a soil which contains impermeable sheets of finite width. The problems are reduced to boundary integral equations which may be solved numerically using established procedures. Numerical results are obtained to provide the distribution of the matric flux potential for some particular combinations of impermeable sheets and a semi-circular channel. The results indicate how the length, number and the depth of impermeable layers influences the distribution of water in an irrigated area.
\end{abstract}

\section{Introduction}

The study of infiltration problems from single or periodic channels has been considered by a number of authors. For example steady infiltration problems of this type have been considered by Philip [1-3], Wooding [4], Raats [5,6], Zachmann and Thomas [7], Pullan and Collins [8] and Batu [9,10] while time dependent problems have been solved by Lomen and Warrick [11] and Warrick [12]. These authors all consider infiltration through a uniform homogeneous soil.

The current study is concerned with the solution of a class of infiltration problems for a homogeneous soil which contains one or more impermeable layers of finite 
width (see Figure 1). A boundary integral formulation is used to facilitate the numerical solution of the governing differential equation and this is then used to determine the effect of the impermeable layers on the distribution of the matric flux potential throughout the soil. The solutions are relevant in assessing the influence of impermeable layers on infiltration from irrigation furrows.

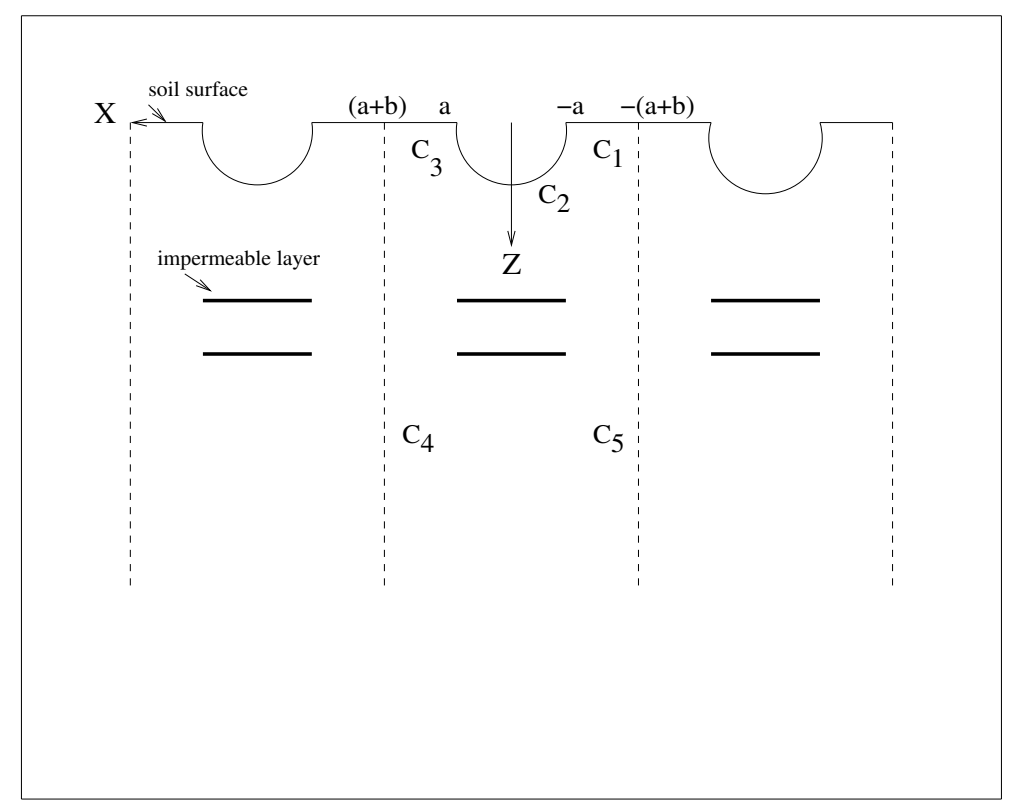

Figure 1: Periodic semi-circular channels with two impermeable layers

\section{Statement of the Problem}

Referred to a Cartesian frame $O X Y Z$ consider an isotropic homogeneous soil lying in the region $Z>0$ with $O Z$ vertically downwards. The region contains a periodic series of semi-circular channels and impermeable layers which have a geometry which does not vary in the $O Y$ direction (see Figure 1). Each of the identical channels has surface area $2 L$ per unit length in the $O Y$ direction where $L$ is a reference length. The spacing between channels is denoted by $2 b$ so that the vertical planes $X= \pm(2 m-1)(a+b)$ for $m=1,2,3, \cdots$ with $a=2 L / \pi$ lie half-way between adjacent channels (see Figure 1 ). The identical channels and impermeable layers are such that the geometry of the region $Z>0$ is symmetrical about the planes $X= \pm m(a+b)$ for $m=0,1,2,3 \cdots$.

The channels are filled with water. The problem is to determine the matric flux potential $\Theta(X, Z)$, the flow throughout the soil in $Z>0$ and the effect of the 
impermeable layers on this flux and flow.

Due to the symmetry of the problem there will be no flow across the planes $X= \pm m(a+b)$ for $m=0,1,2,3, \cdots$ and hence it is sufficient to consider one of the identical regions $(2 m-1)(a+b)<X<(2 m+1)(a+b)$ for $m=0, \pm 1, \pm 2, \pm 3, \cdots$ with a zero normal flow condition across the bounding planes. Attention is therefore restricted to the region $Z>0,|X|<(a+b)$ which will be denoted by $\Omega$ with boundary $\partial \Omega$. In this region there are $N$ plane impermeable layers of finite width which do not intersect with the boundary of the region $\Omega$ or with other impermeable layers (see Figure 1). The $n$th layer is defined to start at coordinates $\left(a^{(n)}, b^{(n)}\right)$ and end at $\left(c^{(n)}, d^{(n)}\right)$. The surface boundary of $\Omega$ along $Z=0$ outside the channel is denoted by $C_{1}$ and $C_{3}$ and the surface of the channel by $C_{2}$ (see Figure 1). The boundary of $\Omega$ along $X=(a+b)$ is denoted by $C_{4}$ and the boundary along $X=-(a+b)$ by $C_{5}$. The union of the surfaces of all the impermeable layers will be referred to as $D$. On $C_{1}$ and $C_{3}$ the normal flow is taken to be zero while a uniform constant normal flow is specified over the surface of the channel. There is zero flow normal to the impermeable layers $D$ and across the boundaries $C_{4}$ and $C_{5}$. Also the derivatives $\partial \Theta / \partial X$ and $\partial \Theta / \partial Z$ vanish as $X^{2}+Z^{2} \rightarrow \infty$.

\section{Fundamental Equations}

The relationship between the hydraulic conductivity $K(h)$ of unsaturated soil and the hydraulic conductivity $K_{s}$ of saturated soil is taken in the exponential form (see Gardner [13] )

$$
K(h)=K_{s} \exp (\alpha h),
$$

where $h$ is the soil water potential and $\alpha$ is an empirical constant that provides a measure of the relative significance of gravity and capillarity for water movement in the soil (see Philip [3] ). The matric flux potential $\Theta$ is related to the hydraulic conductivity by the equation

$$
\Theta=\int_{-\infty}^{h} K(q) d q=\alpha^{-1} K(h) .
$$

The linearized form of the steady infiltration equation is

$$
\frac{\partial^{2} \Theta}{\partial X^{2}}+\frac{\partial^{2} \Theta}{\partial Z^{2}}=\alpha \frac{\partial \Theta}{\partial Z}
$$

The horizontal and vertical components of the flux, as functions of the matric flux potential are

$$
\begin{gathered}
U=-\frac{\partial \Theta}{\partial X}, \\
V=\alpha \Theta-\frac{\partial \Theta}{\partial Z} .
\end{gathered}
$$


The flux normal to a surface with outward pointing normal $\mathbf{n}=\left(n_{1}, n_{2}\right)$ is given by

$$
F=-\frac{\partial \Theta}{\partial X} n_{1}+\left(\alpha \Theta-\frac{\partial \Theta}{\partial Z}\right) n_{2} .
$$

In terms of the dimensionless variables

$$
\begin{array}{r}
\theta=\frac{1}{V_{0} L} \Theta, \quad x=\frac{\alpha}{2} X, \quad z=\frac{\alpha}{2} Z, \\
u=\frac{2}{V_{0} \alpha L} U, \quad v=\frac{2}{V_{0} \alpha L} V, \quad f=\frac{2}{V_{0} \alpha L} F .
\end{array}
$$

where $V_{0}$ is a reference flux, equations (3) to (6) may be written in the dimensionless form

$$
\begin{gathered}
\frac{\partial^{2} \theta}{\partial x^{2}}+\frac{\partial^{2} \theta}{\partial z^{2}}=2 \frac{\partial \theta}{\partial z}, \\
u=-\frac{\partial \theta}{\partial x}, \\
v=2 \theta-\frac{\partial \theta}{\partial z}, \\
f=-\frac{\partial \theta}{\partial x} n_{1}+\left(2 \theta-\frac{\partial \theta}{\partial z}\right) n_{2} .
\end{gathered}
$$

The transformation

$$
\theta=\exp (z) \Psi
$$

transforms equation (8) to the equation

$$
\frac{\partial^{2} \Psi}{\partial x^{2}}+\frac{\partial^{2} \Psi}{\partial z^{2}}-\Psi=0
$$

Also equations (9) to (11) transform to

$$
\begin{gathered}
u=-\exp (z) \frac{\partial \Psi}{\partial x} \\
v=\exp (z)\left(\Psi-\frac{\partial \Psi}{\partial z}\right) \\
f=-\exp (z)\left[\frac{\partial \Psi}{\partial x} n_{1}-\left(\Psi-\frac{\partial \Psi}{\partial z}\right) n_{2}\right] \\
=-\exp (z)\left[\frac{\partial \Psi}{\partial n}-\Psi n_{2}\right] .
\end{gathered}
$$

Hence

$$
\frac{\partial \Psi}{\partial n}=\Psi n_{2}-e^{-z} f
$$

Since the geometry of the problem in Figure 1 is symmetrical about $X= \pm m(a+b)$ for $m=0,1,2,3, \cdots$ the boundary value problem need only be solved for the region 
$-(a+b)<X<(a+b)$ with the following boundary conditions.

There is no flow across $C_{1}$ and $C_{3}$ (see Figure 1) so that from equation (15)

$$
\Psi-\frac{\partial \Psi}{\partial z}=0 \quad \text { for } z=0 \text { and } a \alpha / 2<|x|<(a+b) \alpha / 2 .
$$

Over the surface of the channel the normal flow is

$$
-\left[\frac{\partial \Psi}{\partial x} n_{1}-\left(\Psi-\frac{\partial \Psi}{\partial z}\right) n_{2}\right]=\exp (-z) f_{0}(x, z) \quad \text { for }(x, z) \epsilon C_{2},
$$

where $f_{0}(x, z)$ is given. There is no flow across $C_{4}$ and $C_{5}$ so that from equation (14)

$$
\frac{\partial \Psi}{\partial x}=0 \quad \text { for } z>0 \text { and } x= \pm(a+b) \alpha / 2
$$

Also there is no flow across the impermeable layers so if $\mathbf{n}^{(j)}=\left(n_{1}^{(j)}, n_{2}^{(j)}\right)$ denotes the normal to the $j$ th impermeable layer then for $(x, z)$ on the $j$ th impermeable layer (17) provides

$$
n_{2}^{(j)} \Psi(\xi, \eta)-\frac{\partial \Psi}{\partial n^{(j)}}=0 \quad \text { for } j=1,2, \ldots
$$

The derivatives $\partial \Theta / \partial x$ and $\partial \Theta / \partial z$ are both zero for $z=\infty$ and $0<|x|<$ $(a+b) \alpha / 2$. Hence using (7) and (12) the corresponding conditions on $\Psi$ are

$$
\frac{\partial \Psi}{\partial x}=0 \quad \text { and } \quad \frac{\partial \Psi}{\partial z}=-\Psi \quad \text { for } z=\infty \text { and } 0<|x|<(a+b) \alpha / 2 .
$$

\section{Boundary Integral Equation}

The boundary integral equation for a solution to equation (13) is given by $\Omega$ in $R^{2}$ with

$$
\lambda \Psi(\xi, \eta)=-\int_{\partial \Omega}\left[\frac{\partial \Psi}{\partial n} \phi^{\prime}-\frac{\partial \phi^{\prime}}{\partial n} \Psi\right] d S,
$$

where $\mathbf{n}=\left(n_{1}, n_{2}\right)$ is the outward pointing normal to $\Omega, \lambda=1$ if $(\xi, \eta) \in \Omega$ and $\lambda=1 / 2$ if $(\xi, \eta) \in \partial \Omega$ and $\partial \Omega$ has a continuously turning tangent at $(\xi, \eta)$. The $\phi^{\prime}$ in equation (23) is the fundamental solution of (13) given by

$$
\phi^{\prime}(x, z ; \xi, \eta)=-\frac{1}{2 \pi} K_{0}(r) .
$$

where $r=\left((x-\xi)^{2}+(z-\eta)^{2}\right)^{\frac{1}{2}}$ and $K_{0}$ is the modified Bessel function of order zero. 
Substitution of (17) into (23) gives

$$
\lambda \Psi(\xi, \eta)=-\int_{\partial \Omega}\left[\left(\phi^{\prime} n_{2}-\frac{\partial \phi^{\prime}}{\partial n}\right) \Psi-f e^{-z} \phi^{\prime}\right] d S .
$$

An alternative boundary integral equation formulation which directly relates the potential $\theta$ and the flux $f$ may be obtained as follows. From equation (12)

$$
\Psi(x, z)=e^{-z} \theta(x, z) .
$$

Let

$$
\phi^{\prime \prime}(x, z ; \xi, \eta)=e^{\eta-z} \phi^{\prime}(x, z ; \xi, \eta) .
$$

Then in terms of $\theta$ and $\phi^{\prime \prime}$ the integral equation (25) becomes

$$
\lambda \theta=\int_{\partial \Omega}\left[\frac{\partial \phi^{\prime \prime}}{\partial n} \theta+f \phi^{\prime \prime}\right] d S .
$$

\section{Solution of the Problem}

Equation (25) may be written in the form

$$
\begin{aligned}
\lambda \Psi(\xi, \eta)=-\int_{C \cup D}[ & P(x, z) \phi^{\prime}(x, z ; \xi, \eta) \\
& \left.-\Lambda\left(x, z ; \xi, \eta ; n_{1}, n_{2}\right) \Psi(x, z)\right] d S(x, z),
\end{aligned}
$$

where $C$ is the outer boundary of the body and $D$ denotes the surfaces of the impermeable layers and

$$
\begin{gathered}
P(x, z)=n_{2} \Psi-f e^{-z}, \\
\phi^{\prime}(x, z ; \xi, \eta)=-\frac{1}{2 \pi} K_{0}(r), \\
\Lambda\left(x, z ; \xi, \eta ; n_{1}, n_{2}\right)=\frac{\partial \phi^{\prime}}{\partial x} n_{1}+\frac{\partial \phi^{\prime}}{\partial z} n_{2} \\
=\frac{\left[(x-\xi) n_{1}+(z-\eta) n_{2}\right]}{2 \pi r} K_{1}(r),
\end{gathered}
$$

where $K_{1}(r)$ is the modified Bessel function of order 1 . The outer boundary $C$ in equation (29) is the union of all $C_{s}$ where $s=1,2, \cdots 6$ and $C_{6}$ is the plane $z=k$ where $k$ is constant.

On the $i$ th impermeable layer the coordinates $x$ and $z$ may be written in terms of a single parameter $t$ in the form

$$
\begin{aligned}
& x=X^{(i)}(t)=\left[\left(c^{(i)}-a^{(i)}\right) t+\left(c^{(i)}+a^{(i)}\right)\right] / 2 \text { for } t \in[-1,1], \\
& z=Z^{(i)}(t)=\left[\left(d^{(i)}-b^{(i)}\right) t+\left(b^{(i)}+d^{(i)}\right)\right] / 2 \text { for } t \in[-1,1] .
\end{aligned}
$$


Thus equation (29) may be written in the form

$$
\begin{aligned}
\lambda \Psi(\xi, \eta)= & -\int_{C}\left[P(x, z) \phi^{\prime}(x, z ; \xi, \eta)-\Lambda\left(x, z ; \xi, \eta ; n_{1}, n_{2}\right) \Psi(x, z)\right] d S(x, z) \\
& +\sum_{i=1}^{N} \frac{L^{(i)}}{2} \int_{-1}^{1}\left[\Lambda\left(X^{(i)}(t), Z^{(i)}(t) ; \xi, \eta ; n_{1}^{(i)}, n_{2}^{(i)}\right)\right. \\
& \left.\quad-n_{2}^{(i)} \phi^{\prime}\left(X^{(i)}(t), Z^{(i)}(t) ; \xi, \eta\right)\right] \Delta w^{(i)}(t) d t, \quad
\end{aligned}
$$

where $L^{(i)}$ is the length of the $i$ th impermeable layer, $\left(n_{1}^{(i)}, n_{2}^{(i)}\right)=$ $\left(\left[d^{(i)}-b^{(i)}\right] / L^{(i)},-\left[c^{(i)}-a^{(i)}\right] / L^{(i)}\right)$ and $\Delta w^{(i)}(t)=\Psi\left(X^{(i)}(t), Z^{(i)}(t)^{+}\right)-$ $\Psi\left(X^{(i)}(t), Z^{(i)}(t)^{-}\right)$gives the jump in $\Psi$ across the opposite faces of the $i$ th impermeable layer.

It is convenient at this point to define $\Phi$ and $\Omega$ as follows.

$$
\begin{array}{r}
\Phi^{(i)}\left(x, z ; \xi, \eta ; n_{1}^{(i)}, n_{2}^{(i)}\right)=\frac{\partial \phi^{\prime}}{\partial n^{(i)}}=\frac{\partial \phi^{\prime}}{\partial \xi} n_{1}^{(i)}+\frac{\partial \phi^{\prime}}{\partial \eta} n_{2}^{(i)} \\
=-\frac{\left[(x-\xi) n_{1}^{(i)}+(z-\eta) n_{2}^{(i)}\right]}{2 \pi r} K_{1}(r), \\
\Omega^{(i)}\left(x, z ; \xi, \eta ; n_{1}, n_{2} ; n_{1}^{(i)}, n_{2}^{(i)}\right)=\frac{\partial \Lambda}{\partial n^{(i)}}=\frac{\partial \Lambda}{\partial \xi} n_{1}^{(i)}+\frac{\partial \Lambda}{\partial \eta} n_{2}^{(i)} \\
=-\frac{1}{2 \pi r}\left[n_{1} n_{1}^{(i)}+n_{2} n_{2}^{(i)}\right] K_{1}(r) \\
+\frac{\left[(x-\xi) n_{1}+(z-\eta) n_{2}\right]\left[(x-\xi) n_{1}^{(i)}+(z-\eta) n_{2}^{(i)}\right]}{2 \pi r^{3}}\left[2 K_{1}(r)+r K_{0}(r)\right] .
\end{array}
$$

Now as $(\xi, \eta)$ approaches the $i$ th impermeable layer the integral over this impermeable layer in (35) must be interpreted as a Cauchy principal value integral. Hence differentiation of this integral (with respect to either $\xi$ or $\eta$ ) as $(\xi, \eta)$ approaches the impermeable layer leads to a Hadamard finite-part integral.

On the $j$ th impermeable layer the coordinates $\xi$ and $\eta$ may be written in terms of a single parameter $s$ in the form

$$
\begin{aligned}
& \xi=X^{(j)}(s)=\left[\left(c^{(j)}-a^{(j)}\right) s+\left(c^{(j)}+a^{(j)}\right)\right] / 2 \text { for } s \in[-1,1], \\
& \eta=Z^{(j)}(s)=\left[\left(d^{(j)}-b^{(j)}\right) s+\left(b^{(j)}+d^{(j)}\right)\right] / 2 \text { for } s \in[-1,1] .
\end{aligned}
$$

Thus using equations (35) the zero flux condition (21) on the $j$ th impermeable 
layer may be expressed as

$$
\begin{aligned}
& \lambda\left[n_{2}^{(j)} \Psi(\xi, \eta)-\frac{\partial \Psi}{\partial n^{(j)}}\right] \\
= & -n_{2}^{(j)} \int_{C}\left[P(x, z) \phi^{\prime}(x, z ; \xi, \eta)-\Lambda\left(x, z ; \xi, \eta ; n_{1}, n_{2}\right) \Psi(x, z)\right] d S(x, z) \\
& +n_{2}^{(j)} \sum_{i=1}^{N} \frac{L^{(i)}}{2} \int_{-1}^{1}\left[\Lambda\left(X^{(i)}(t), Z^{(i)}(t) ; \xi, \eta ; n_{1}^{(i)}, n_{2}^{(i)}\right)\right. \\
& \left.\quad-n_{2}^{(i)} \phi^{\prime}\left(X^{(i)}(t), Z^{(i)}(t) ; \xi, \eta\right)\right] \Delta w^{(i)}(t) d t \\
& \int_{C}\left[P(x, z) \Phi^{(j)}\left(x, z ; \xi, \eta ; n_{1}^{(j)}, n_{2}^{(j)}\right)\right. \\
& -\sum_{i=1}^{N} \frac{L^{(i)}}{2} \int_{-1}^{1}\left[\Omega^{(j)}\left(X^{(i)}(t), Z^{(i)}(t) ; \xi, \eta ; n_{1}^{(i)}, n_{2}^{(i)} ; n_{1}^{(j)}, n_{2}^{(j)}\right)\right. \\
= & 0 .
\end{aligned}
$$

If $(\xi, \eta)$ is on $L^{(j)}$ then on the $L^{(j)}$ th impermeable layer it follows from (33), (34), (38) and (39) together with the expressions for the normal components on the layer that

$$
\begin{aligned}
& \Lambda\left(X^{(j)}(t), Z^{(j)}(t) ; X^{(j)}(s), Z^{(j)}(s) ; n_{1}^{(j)}, n_{2}^{(j)}\right)=0, \\
& \Phi^{(j)}\left(X^{(j)}(t), Z^{(j)}(t) ; X^{(j)}(s), Z^{(j)}(s) ; n_{1}^{(j)}, n_{2}^{(j)}\right)=0, \\
& \Omega^{(j)}\left(X^{(j)}(t), Z^{(j)}(t) ; X^{(j)}(s), Z^{(j)}(s) ; n_{1}^{(j)}, n_{2}^{(j)} ; n_{1}^{(j)}, n_{2}^{(j)}\right)=-\frac{1}{2 \pi r} K_{1}(r) \\
& =\frac{-1}{2 \pi}\left[\frac{1}{r^{2}}+\frac{1}{2} \ln r-R(r)\right],
\end{aligned}
$$

where the formulas [9.8.3] and [9.8.7] in Abramowitz and Stegun [14] may be used 
to obtain $R(r)$ in the form

$$
\begin{array}{ll}
R(r) & =-1 / 2 \log (2)+\log (r / 2) r^{2}\left[0.87890594\left(\frac{1}{3.75^{2}}\right)\right. \\
+ & 0.51498869\left(\frac{1}{3.75^{4}}\right) r^{2}+0.15084934\left(\frac{1}{3.75^{6}}\right) r^{4} \\
+ & 0.02658733\left(\frac{1}{3.75^{8}}\right) r^{6}+0.00301532\left(\frac{1}{3.75^{10}}\right) r^{8} \\
+ & \left.0.00032411\left(\frac{1}{3.75^{12}}\right) r^{10}\right]+0.15443144(1 / 4)-0.67278579\left(r^{2} / 16\right) \\
- & 0.18156897\left(r^{4} / 32\right)-0.01919402\left(r^{6} / 64\right)-0.00110404\left(r^{8} / 128\right) \\
- & 0.00004686\left(r^{10} / 256\right), \quad \text { where } 0<r \leq 2
\end{array}
$$

Hence if $(\xi, \eta)$ is on $L^{(j)}$ then equation (40) may be written in the form

$$
\begin{aligned}
& -\quad n_{2}^{(j)} \int_{C}\left[P(\mathbf{x}) \phi^{\prime}\left(\mathbf{x} ; \mathbf{X}^{(j)}(s)\right)-\Lambda\left(\mathbf{x} ; \mathbf{X}^{(j)}(s) ; \mathbf{n}\right) \Psi(\mathbf{x})\right] d S(\mathbf{x}) \\
& +n_{2}^{(j)} \sum_{i \neq j} \frac{L^{(i)}}{2} \int_{-1}^{1}\left[\Lambda\left(\mathbf{X}^{(i)}(t) ; \mathbf{X}^{(j)}(s) ; \mathbf{n}^{(i)}\right)\right. \\
& \left.-n_{2}^{(i)} \phi^{\prime}\left(\mathbf{X}^{(i)}(t) ; \mathbf{x}_{0}\right)\right] \Delta w^{(i)}(t) d t \\
& -\quad\left(n_{2}^{(j)}\right)^{2} \frac{L^{(j)}}{2} \int_{-1}^{1}\left[\phi^{\prime}\left(\mathbf{X}^{(j)}(t) ; \mathbf{X}^{(j)}(s)\right)\right] \Delta w^{(j)}(t) d t \\
& +\int_{C}\left[P(\mathbf{x}) \Phi^{(j)}\left(\mathbf{x} ; \mathbf{X}^{(j)}(s) ; \mathbf{n}^{(j)}\right)\right. \\
& -\sum_{i \neq j} \frac{L^{(i)}}{2} \int_{-1}^{1}\left[\Omega^{(j)}\left(\mathbf{X}^{(i)}(t) ; \mathbf{X}^{(j)}(s) ; \mathbf{n}^{(i)} ; \mathbf{n}^{(j)}\right)\right. \\
& +\quad \frac{1}{\pi L^{(j)}} \mathcal{H} \int_{-1}^{1} \frac{\Delta w^{(j)}(t)}{(t-s)^{2}} d t+\frac{L^{(j)}}{8 \pi} \int_{-1}^{1} \log \left(\frac{L^{(j)}}{2}|t-s|\right) \Delta w^{(j)}(t) d t \\
& \quad-\frac{L^{(j)}}{4 \pi} \int_{-1}^{1} R\left(\frac{L^{(j)}}{2}|t-s|\right) \Delta w^{(j)}(t) d t \\
& =0, \quad \text { for } \quad-1<s<1,
\end{aligned}
$$

where $\mathcal{H}$ denotes the Hadamard finite-part integral and

$$
\mathbf{x}=(x, z), \quad \mathbf{x}_{0}=(\eta, \zeta), \quad \mathbf{X}^{(i)}=\left(X^{(i)}, Z^{(i)}\right) .
$$


The boundary integral equations (35) and (45) form the basis of a boundary element procedure for the solution of the boundary value problem.

Let

$$
\Delta w^{(i)}(t) \simeq \sqrt{1-t^{2}} \sum_{k=1}^{J^{(i)}} \alpha_{k}^{(i)} U_{k-1}(t)
$$

where $U_{k}(t)$ denotes the Chebyshev polynomial of the second kind and the $\alpha_{k}^{(i)}$ for $i=1 \ldots N$ and $k=1 \ldots J^{(i)}$ are unknown constants.

The boundary $C$ is approximated by $M$ straight line segments $\mathcal{C}_{(q)}(q=1,2 \ldots M)$ of equal length so that

$$
C \simeq \mathcal{C}_{(1)} \cup \mathcal{C}_{(2)} \cup \cdots \cup \mathcal{C}_{(M)},
$$

On the $q$ th segment the functions $\Psi$ and $f$ are taken to be constant so that

$$
\Psi \simeq \Psi^{(q)} \text { constant over } \mathcal{C}_{(q)}, \quad f \simeq f^{(q)} \text { constant over } \mathcal{C}_{(q)}
$$

Letting $\left(\xi^{(m)}, \eta^{(m)}\right)$ be the midpoint of $C_{m}$, the equation (35) may be approximated by

$$
\begin{aligned}
& \lambda \Psi^{(m)}= \sum_{q=1}^{M}\left\{f^{(q)} \int_{\mathcal{C}_{(q)}} e^{-z} \phi^{\prime}\left(\mathbf{x} ; \mathbf{x}_{\mathbf{0}}{ }^{(m)}\right) d S(\mathbf{x})\right. \\
&\left.+\Psi^{(q)} \int_{\mathcal{C}_{(q)}}\left[\Lambda\left(\mathbf{x} ; \mathbf{x}_{\mathbf{0}}{ }^{(m)} ; \mathbf{n}\right)-n_{2} \phi^{\prime}\left(\mathbf{x} ; \mathbf{x}_{\mathbf{0}}{ }^{(m)}\right)\right] d S(\mathbf{x})\right\} \\
&+\sum_{i=1}^{N} \frac{L^{(i)}}{2} \sum_{k=1}^{J^{(i)}} \alpha_{k}^{(i)} \int_{-1}^{1}\left[\Lambda\left(\mathbf{X}^{(i)}(t) ; \mathbf{x}_{\mathbf{0}}{ }^{(m)} ; \mathbf{n}^{(i)}\right)\right. \\
&\left.-n_{2}^{(i)} \phi^{\prime}\left(\mathbf{X}^{(i)}(t) ; \mathbf{x}_{\mathbf{0}}{ }^{(m)}\right)\right] U_{k-1}(t) \sqrt{1-t^{2}} d t, \\
& \text { for } m=1,2, \ldots, M .
\end{aligned}
$$

The discretised formulation of equation (45) can be obtained in a similar manner. 
Replacing $P(x, z)$ with $n_{2} \Psi-f e^{-z}$ then equation (45) can be approximated by

$$
\begin{aligned}
& \sum_{q=1}^{M}\left\{f^{(q)} \int_{\mathcal{C}_{(q)}} e^{-z}\left[n_{2}^{(j)} \phi^{\prime}\left(\mathbf{x} ; \mathbf{X}^{(j)}(s)\right)-\Phi^{(j)}\left(\mathbf{x} ; \mathbf{X}^{(j)}(s) ; \mathbf{n}^{(j)}\right)\right] d S(\mathbf{x})\right. \\
& +\Psi^{(q)} \int_{\mathcal{C}_{(q)}}\left[n _ { 2 } ^ { ( j ) } \left(\Lambda\left(\mathbf{x} ; \mathbf{X}^{(j)}(s) ; \mathbf{n}\right)\right.\right. \\
& -n_{2} \phi^{\prime}\left(\mathbf{x} ; \mathbf{X}^{(j)}(s)\right)+n_{2} \Phi^{(j)}\left(\mathbf{x} ; \mathbf{X}^{(j)}(s) ; \mathbf{n}^{(j)}\right) \\
& \left.\left.-\Omega^{j}\left(\mathbf{x} ; \mathbf{X}^{(j)}(s) ; \mathbf{n} ; \mathbf{n}^{(j)}\right)\right] d S(\mathbf{x})\right\} \\
& +n_{2}^{(j)} \sum_{i \neq j} \frac{L^{(i)}}{2} \sum_{k=1}^{J^{(i)}} \alpha_{k}^{(i)} \int_{-1}^{1}\left[\Lambda\left(\mathbf{X}^{(i)}(t) ; \mathbf{X}^{(j)}(s) ; \mathbf{n}^{(i)}\right)\right. \\
& -\left(n_{2}^{(j)}\right)^{2} \frac{L^{(j)}}{2} \sum_{k=1}^{J^{(j)}} \alpha_{k}^{(j)} \int_{-1}^{1}\left[\phi^{\prime}\left(\mathbf{X}^{(j)}(t) ; \mathbf{X}^{(j)}(s)\right)\right] U_{k-1}(t) \sqrt{1-t^{2}} d t \\
& -\sum_{i \neq j} \frac{L^{(i)}}{2} \sum_{k=1}^{J^{(i)}} \alpha_{k}^{(i)} \int_{-1}^{1}\left[\Omega^{(j)}\left(\mathbf{X}^{(i)}(t) ; \mathbf{X}^{(j)}(s) ; \mathbf{n}^{(i)} ; \mathbf{n}^{(j)}\right)\right. \\
& \left.-n_{2}^{(i)} \Phi^{(j)}\left(\mathbf{X}^{(i)}(t) ; \mathbf{X}^{(j)}(s)\right)\right] U_{k-1}(t) \sqrt{1-t^{2}} d t \\
& +\frac{1}{\pi L^{(j)}} \sum_{k=1}^{J^{(j)}} \alpha_{k}^{(j)} \mathcal{H} \int_{-1}^{1} \frac{U_{k-1}(t) \sqrt{1-t^{2}}}{(t-s)^{2}} d t \\
& +\frac{L^{(j)}}{8 \pi} \sum_{k=1}^{J^{(j)}} \alpha_{k}^{(j)} \int_{-1}^{1} \log \left(\frac{L^{(j)}}{2}|t-s|\right) U_{k-1}(t) \sqrt{1-t^{2}} d t \\
& -\frac{L^{(j)}}{4 \pi} \sum_{k=1}^{J^{(j)}} \alpha_{k}^{(j)} \int_{-1}^{1} R\left(\frac{L^{(j)}}{2}|t-s|\right) U_{k-1}(t) \sqrt{1-t^{2}} d t \\
& =0, \text { for }-1<s<1 \text { and } j=1,2, \ldots, N .
\end{aligned}
$$

The Hadamard finite-part integral may be evaluated using the formula (see Kaya and Erdogan [15] )

$$
\mathcal{H} \int_{-1}^{1} \frac{U_{k-1}(t) \sqrt{1-t^{2}}}{(t-s)^{2}} d t=-\pi k U_{k-1}(s)
$$

For the purpose of numerical calculations the number of constants $\alpha_{k}^{(i)}$ is taken to be the same for each impermeable layer so that $J^{(i)}=J$ say for $i=1,2, \cdots, N$. Hence equation (47) consists of $M$ equations (since $m=1,2, \ldots, M$ ) in the $M+N J$ unknowns $\Psi_{m}$ for $m=1,2, \ldots, M$ and $\alpha_{k}^{(j)}$ for $k=1,2, \ldots, J$ and $j=1,2, \ldots, N$. 
In order to generate the extra $N J$ equations required to solve the system equation (48) is employed. Specifically, in (48) $J$ points are taken on each impermeable layer (for instance setting $s=s_{p}=\cos ([2 p-1] \pi /[2 J]),(p=1,2, \ldots, J)$ for each layer) so that (48) generates a linear system of $N J$ equations for the unknowns $\Psi_{m}$ for $m=1,2, \ldots, M$ and $\alpha_{k}^{(j)}$ for $k=1,2, \ldots, J$ and $j=1,2, \ldots, N$. Thus the total number of unknowns $N J+M$ is equal to the number of linear algebraic equations and the unknowns can be determined.

\section{Numerical Results}

In this section numerical results are given for the values of the matric flux potential associated with infiltration from a semi-circular channel of radius $a=2 L / \pi$ with one or more impermeable layers located in various positions in the domain $Z>$ $0,|X|<(a+b)$ (see Figure 1). The normal flux over the surface of the channel is chosen to be constant $F=-V_{0}$. From the definition of the dimensionless variables in equation (7) and using the value of $\alpha$ given by Philip [3] (that is $\alpha=0.002 \mathrm{~cm}^{-1}$ ) and letting the reference length $L=100 \mathrm{~cm}$ it follows that $\alpha L=0.2$ and therefore

$$
f=f_{0}=\frac{2}{0.2 V_{0}}(-F)=-10 .
$$

Also the dimensionless radius $a_{1}$ of the semi-circular channel is given by

$$
a_{1}=\frac{\alpha}{2} a=\frac{2 \alpha L}{2 \pi}=0.2 / \pi .
$$

Thus the total non-dimensional inflow of water through the surface of the semicircular channel is given by

$$
\pi a_{1} f=-2 .
$$

This inflow must exactly match the outflow across the boundary $C_{6}$ which is given by $z=k$ for $|x|<0.5$.

The value of the dimensionless quantity $(a+b) \alpha / 2$ (see Figure 1 ) was taken to be 0.5 (see Figure 2).

The boundary integral equations (47) and (48) and the transformation in equation (12) were used to calculate the dimensionless values of $\theta$ along the boundary line $x=0.5$. The outer boundary $C$ was divided into segments to facilitate the replacement of the integrals in (47) and (48) by a sum to form a system of linear algebraic equations for the unknown function $\Psi(a, b)$.

The results were then transformed to $\theta(x, z)$ using the transformation given by equation (12). The number of segments and the value of $k$ were increased until convergence of the value of $\theta(x, z)$ (to four decimal places) was achieved. To 


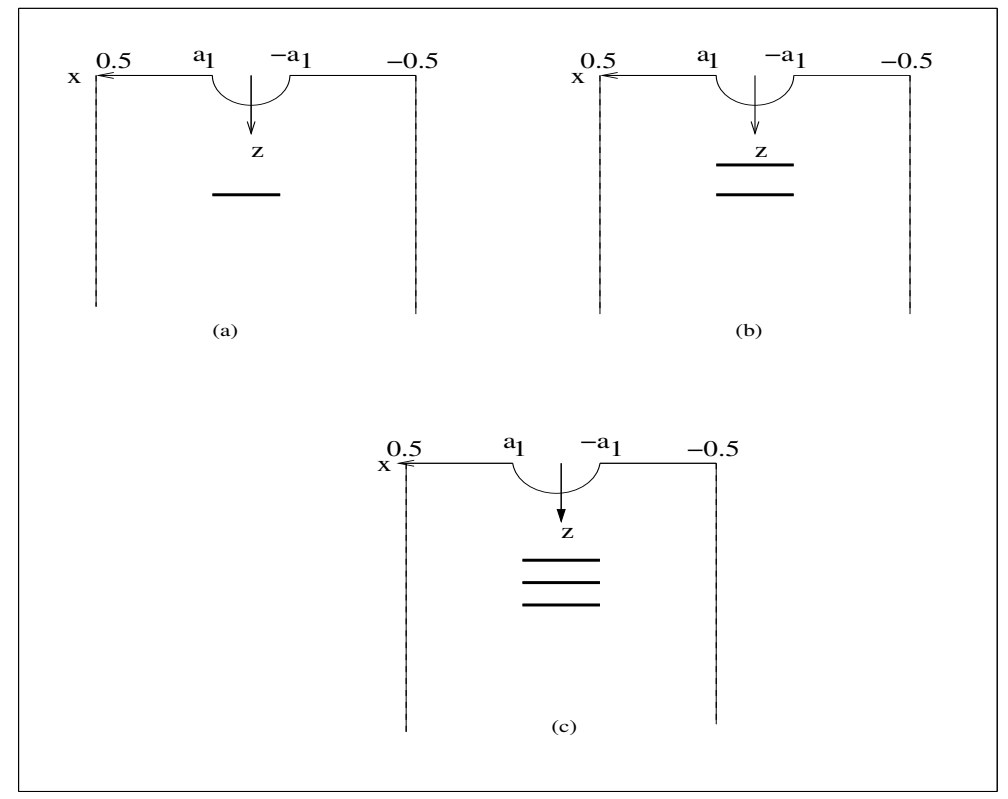

Figure 2: Semi-circular strip source with impermeable layer(s) $\left(a_{1}=0.2 / \pi\right)$

obtain this level of convergence it was necessary to take the value $k=4$ (using the condition $\partial \Psi / \partial z=-\Psi$ on $z=4$ ) and to divide the outer boundary $C$ into 600 segments and also to set $J=6$ in equation (48).

Figure 3 illustrates the dimensionless value of the matric flux potential as a function of dimensionless depth of a single impermeable layer. The values were obtained for a single layer varying in length. In the figure $L_{q}$ denotes the layer of length $q$ lying between the coordinates $(-q / 2,0.25)$ and $(q / 2,0.25)$.

The results indicate clearly that the peak increases with the length of the impermeable layer. This is to be expected, since the longer the impermeable layer, the bigger the capillarity effects relative to those from gravity. Consequently more flux is forced to flow horizontally towards the boundary.

Figure 4 provides the dimensionless value of the matric flux potential for a single impermeable layer placed at different distances below the soil surface. $L_{z}$ denotes the impermeable layer lying between the coordinates $(-0.3, z)$ and $(0.3, z)$. From the graphs it is apparent that, as the depth of the layer below the surface increases, the maximum value of the matric flux potential on the line $x=0.5$ increases but the maximum occurs further below the soil surface. 
Matric Flux

Potential $\theta(0.5, z)$

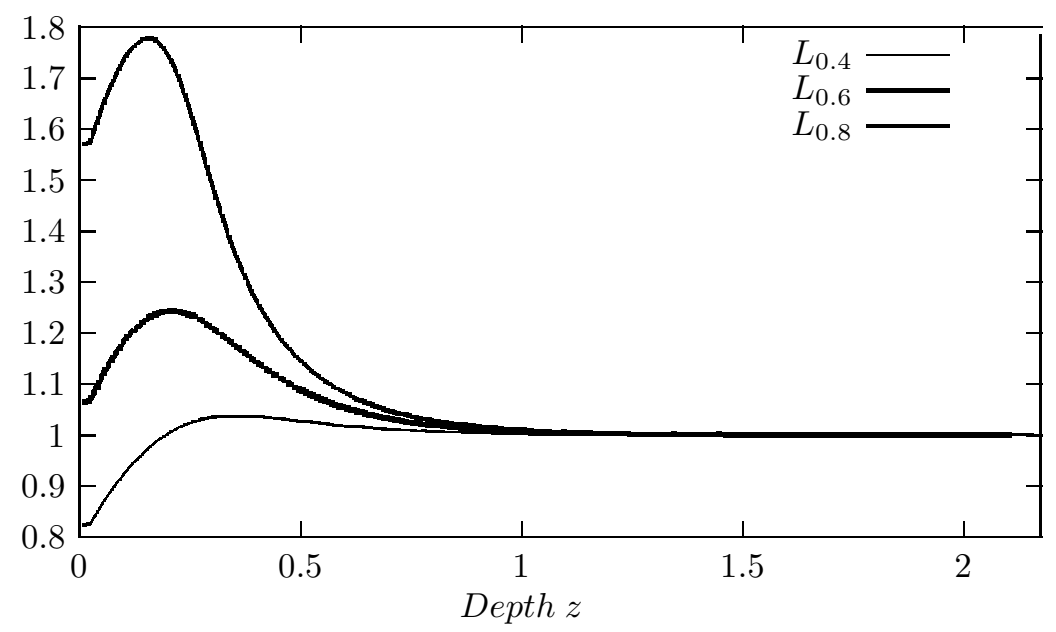

Figure 3: Values of $\theta$ for a single layer varying in length Matric Flux

Potential $\theta(0.5, z)$

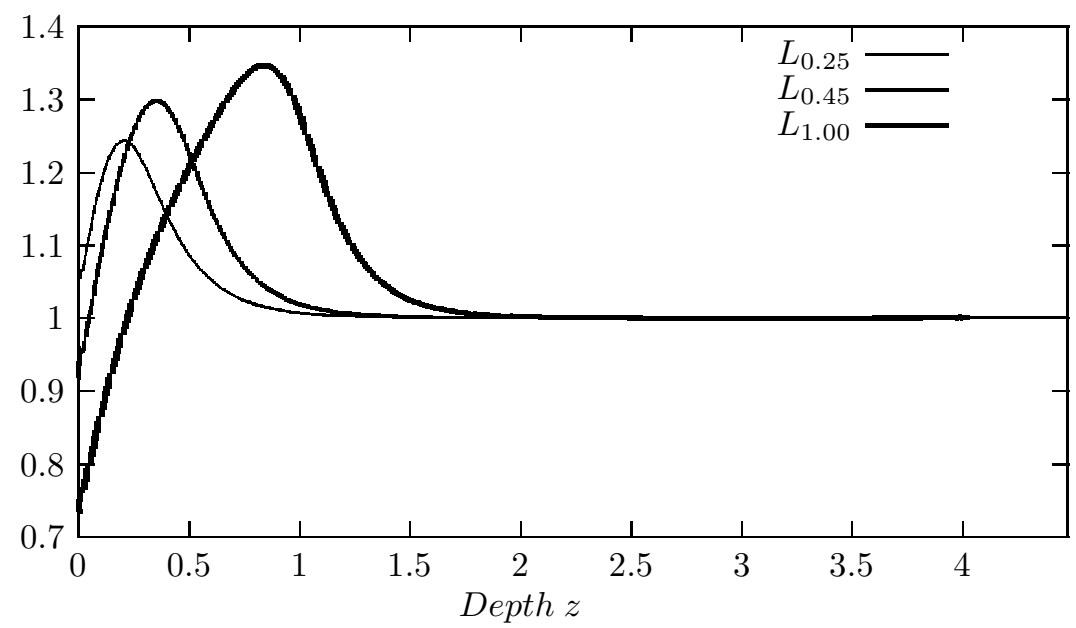

Figure 4: Values of $\theta$ for a single layer varying in depth

The effect of increasing the number of impermeable layers on the matric flux potential is illustrated in Figure 5. In the figure $L_{1}$ provides the matric flux potential for a single layer lying between $(-0.3,0.25)$ and $(0.3,0.25), L_{2}$ provides the potential for two layers with the first layer lying between $(-0.3,0.25)$ and $(0.3,0.25)$ and second layer lying between $(-0.3,1)$ and $(0.3,1)$ (see Figure $2 b)$. $L_{3}$ provides the potential for three layers with the first layer lying between $(-0.3$, 


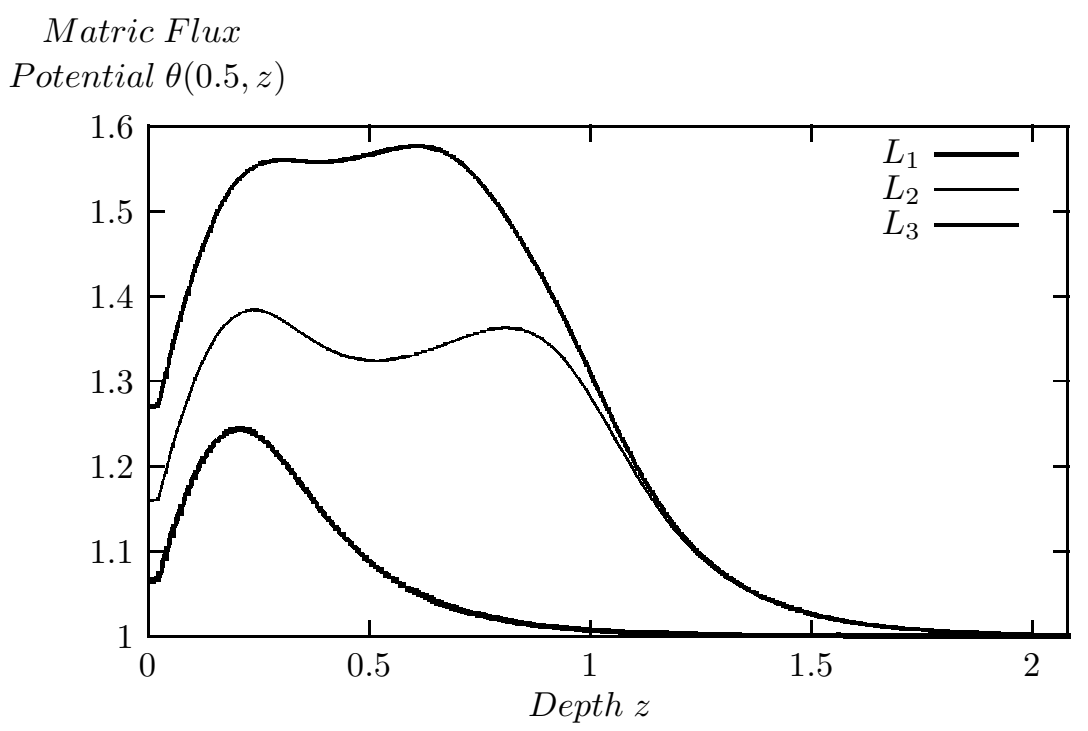

Figure 5: Values of $\theta$ for one, two and three layers

$0.25)$ and $(0.3,0.25)$, the second layer lying between $(-0.3,0.75)$ and $(0.3,0.75)$ and the third layer lying between $(-0.3,1)$ and $(0.3,1)$ (see Figure $2 \mathrm{c})$. The graph indicates how the introduction of the additional impermeable layers provides a higher sustained level of matric flux potential along the line $x=0.5$.

\section{Summary}

A boundary element method for calculating the matric flux potential for infiltration from periodic irrigation channels in a in homogeneous soil with a finite number of impermeable layers has been developed. The method has been employed to determine the effect of impermeable layers on the distribution of the matric flux potential throughout the soil. The results illustrate how the matric flux potential can be influenced by an increase in the length of an impermeable layer. The results also indicate how the depth of the impermeable layers can influence the distribution of the matric flux potential.

\section{References}

[1] J. R. Philip, Theory of infiltration. Adv. Hydrosci. 5, 215-296, (1971).

[2] J. R. Philip, General theorem on steady infiltration from surface sources with application to point and line sources. Soil Science Society of America Proceedings. 35, 867-871, (1971).

[3] J. R. Philip, Steady infiltration from burried point sources and spherical cavities. 
Water Resources Research. 4, 1039-1047, (1968).

[4] R. A. Wooding, Steady infiltration from shallow circular pond. Water Resour. Res. 4, 1259-1273, (1968).

[5] P. A. C. Raats, Steady infiltration from point sources, cavities and basins. Soil Science Society of America Proceedings. 35, 689-694, (1971).

[6] P. A. C. Raats, Steady infiltration from line sources and furrows. Soil Science Society of America Proceedings. 34, 709-714, (1970).

[7] D. W. Zachman and A. W. Thomas, A mathematical investigation of steady infiltration from line sources. Soil Science Society of America Proceedings. 37, 495-500, (1973).

[8] A. J. Pullan and I. F. Collins, Two- and three-dimensional steady quasi-linear infiltration from buried surface cavities using boundary element techniques. Water Resources Research. 23, 1633-1644, (1987).

[9] V. Batu, Steady infiltration from single and periodic strip sources. Soil Science Society of America Journal. 42, 545-549, (1978).

[10] V. Batu, Steady infiltration from a ditch : theory and experiment. Soil Science Society of America Journal. 41, 677-682, (1977).

[11] D. O. Lomen and A. W. Warrick, Time dependent linearized infiltration. II. Line sources. Soil Science Society of America Proceedings. 38. 568-572, (1974).

[12] A. W. Warrick, Time-dependent linearized infiltration: I. Point sources. Soil Science Society of America Proceedings. 38, 383-386, (1974).

[13] W. R. Gardner, Some steady state solutions of the unsaturated moisture flow equation with application to evaporation from a water table. Soil Science. 85, 228-232, (1957).

[14] M. Abramowitz and I. A. Stegun, Handbook of Mathematical Functions, Dover, New York (1972).

[15] A. C. Kaya and F. Erdogan, On the solution of integral equations with strongly singular kernels, Quart. Appl. Maths. 45, 105-122, (1987). 